\title{
Author Correction: Primary Metabolism Co-Opted for Defensive Chemical Production in the Carabid Beetle, Harpalus pensylvanicus
}

\author{
Adam M. Rork ${ }^{1} \cdot$ Sihang $\mathrm{Xu}^{2} \cdot$ Athula Attygalle $^{2} \cdot$ Tanya Renner $^{1}$ \\ Published online: 31 March 2021 \\ (C) Springer Science+Business Media, LLC, part of Springer Nature 2021
}

\section{Author Correction: Journal of Chemical Ecology. https://doi.org/10.1007/s10886-021-01253-2}

The original version of this article unfortunately contained a mistake. In the Methods section "Transcriptome Assembly and Quality Control", the sentence: "With the exception of read orientation being specified as "RF", default settings were used for assembly including in silico read depth normalization which removed reads with coverage $>200$." should read as, "With the exception of read orientation being specified as "RF" and minimum contig length set to $300 \mathrm{bp}$ instead of $200 \mathrm{bp}$, default settings were used for assembly. This includes in silico read depth normalization which removed reads with coverage >200."

In the Methods section "Phylogenetic Reconstruction of Metazoan MTHFD Gene Family", the sentence:
"Phylogenetic model selection was performed with ModelFinder as part of the IQ-Tree package (v1.6.12), which recovered LG + R6 as the best-fit model of the 542 tested (Kalyaanamoorthy et al. 2017; Nguyen et al. 2015)." should read as, "Phylogenetic model selection was performed with ModelFinder as part of the IQ-Tree package (v1.6.12), which recovered $\mathrm{LG}+\mathrm{I}+\mathrm{G} 4$ as the best-fit model of those tested (Kalyaanamoorthy et al. 2017; Nguyen et al. 2015)."

The occurrences of the phrases "black and white should be used for these figures" and "colors should be used for this/ these figure(s)" in the figure descriptions were originally intended as notes to the editorial staff. Their inclusion in the final article was unintentional.

The original article has been corrected.

The online version of the original article can be found at https://doi.org/ 10.1007/s10886-021-01253-2

Adam M. Rork

amr483@psu.edu

Sihang Xu

sxu4@stevens.edu

Athula Attygalle

athula.attygalle@stevens.edu

Tanya Renner

tur158@psu.edu

1 Department of Entomology, The Pennsylvania State University, 501 ASI Building, University Park, PA 16802, USA

2 Department of Chemistry and Chemical Biology, Stevens Institute of Technology, Hoboken, NJ 07030, USA 